\title{
Intakes and sources of dietary sugars and their association with metabolic and inflammatory markers: the Fenland Study, UK
}

\author{
L. O’Connor $^{1,2}$, F. Imamura ${ }^{1}$, S. Brage ${ }^{1}$, S.J. Griffin ${ }^{1}$, N.J. Wareham ${ }^{1}$ and N.G. Forouhi ${ }^{1}$ \\ ${ }^{1}$ MRC Epidemiology Unit, University of Cambridge School of Clinical Medicine, Institute of Metabolic Science, \\ Cambridge Biomedical Campus, Cambridge, CB2 OQQ and ${ }^{2}$ Food and Nutrition Research Cluster, Hollings Faculty, \\ Manchester Metropolitan University, Manchester, M15 6BH
}

It is widely accepted that dietary sugars promote adverse metabolic outcomes via weight-gain through their contribution to energy intake. Emerging evidence suggests that dietary sugars are associated with adverse metabolic outcomes including increased blood pressure and serum lipids, independently of energy intake and body-weight ${ }^{(1)}$. However, dietary sugars are a complex component of the diet and their effects on health outcomes are likely to differ depending on the properties of the consumed sugars. Therefore, associations between dietary sugars and metabolic and inflammatory markers may vary according to whether sugars are from beverage or food sources and, extrinsic or intrinsic.

The aim of this study was to examine the association between intakes of dietary sugars from different sources and metabolic and inflammatory markers using a population-based cross-sectional study of adults in the East of England. Sugar intakes from beverages (liquids), foods (solids), extrinsic (free sugars) or intrinsic (non-free sugars) were estimated using food frequency questionnaires. Glycated haemoglobin, glucose, insulin, and C-Reactive Protein (CRP) were measured in fasting blood samples and insulin resistance (HOMA-IR) and a continuous metabolic risk z-score were derived.

The contributions to total energy intake (TEI) of sugars from liquids, solids, free sugars and non-free sugars contributed $2.9 \%, 20.0 \%$, $12.0 \%$ and $11.6 \%$ respectively. In multiple linear regression analyses adjusted for age, sex, socio-economic status, smoking status, alcohol consumption, physical activity, clinical history, BMI, TEI, dietary quality, fat intake and sugars from other sources, and corrected for multiple testing, sugars from liquids (per $10 \% \mathrm{TEI})$ were positively associated with $\ln -\mathrm{CRP}[\beta$-coefficient $(95 \% \mathrm{CI}), 0 \cdot 14(0 \cdot 05,0 \cdot 22)]$ and the metabolic risk z-score $[0 \cdot 13(0 \cdot 07,0 \cdot 18)]$. Free sugars were positively associated with ln-HOMA-IR $[0 \cdot 05(0 \cdot 03,0 \cdot 08)]$ and the metabolic risk z-score $[0 \cdot 09(0 \cdot 06,0 \cdot 12)]$. Sugars from solids were not associated with any outcome. Among major food and beverage contributors to intake, sugars (per $10 \%$ TEI) in fruit, vegetables, dairy products/egg dishes, cakes/biscuits/confectionary and fruit squash/juice drinks were not associated, but sugar added to tea, coffee, cereal was significantly positively associated with all outcomes. Sugars in 100 $\%$ fruit juice $[0 \cdot 16(0 \cdot 06,0 \cdot 25)]$ and other non-alcoholic beverages $[0 \cdot 13(0 \cdot 03,0 \cdot 23)]$ and metabolic risk z-score were positively associated.

In conclusion, higher intakes of sugars from non-alcoholic beverages and sugar added to tea, coffee, cereal were associated with glycaemia and inflammatory markers. Sugars from solids were not associated, irrespective of whether they were intrinsic or extrinsic. The positive associations of free sugars were largely explained by the contribution of beverages to intake. Adverse metabolic consequences of sugar intake, independent of contribution to caloric intake, may be attributable to sugar intake from beverages.

The Fenland Study is funded by the Wellcome Trust and the Medical Research Council. Support from Medical Research Council programmes MC_UU_12015/1 and MC_UU_12015/5 is acknowledged.

1. Te Morenga L, Mallard S Mann J (2013) BMJ 346, e7492. 